\title{
PSV-FLC Fabricated Using Photo-Alignment Technique
}

\author{
H. Furue, ${ }^{1, *}$ T. Shime, ${ }^{1}$ Y. Ebato, ${ }^{1}$ S. Oka, ${ }^{2}$ S. Komura, ${ }^{2}$ and S. Kobayashi ${ }^{3}$ \\ 1 Department of Materials Science and Technology, Tokyo University of Science, Katsushika, Tokyo, Japan \\ 2 R\&D Division, Japan Display, Mobara, Chiba, Japan \\ 3 Liquid Crystal Institute, Tokyo University of Science, Yamaguchi, Sanyoonoda, Yamaguchi, Japan
}

Polymer-stabilized ferroelectric liquid crystals (PS-FLCs), in which a photocurable mesogenic monomer is doped into an FLC and UV photociring is carried out in the SmA phase at the quiescent condition, may show monostable and $\mathrm{V}$-shaped electrooptical characteristics with a grayscale capability without a threshold.[1, 2] The PS-FLCs with V-shaped electrooptical characteristics are usually called PSV-FLCs. PSV-FLCs are attractive for a next generation of LC display having high performances such as high-quality moving video image and very low power consumption, because of their unique characteristics such as high-speed response and wide viewing angle. However, there is a fundamental problem for their display application. Although the rubbing technique is conventionally used for the LC molecular alignment, the rubbing scratches form on the alignment film surface. In the case of nematic $\mathrm{LC}$, the rubbing scratch pattern of the LC molecular alignment texture fall into obscurity to some extent because of relatively easy self-assembly of the molecular orientation. On the other hand, the smectic LC forms a layer structure of the molecular alignment, and is relatively similar to crystal. Therefore, the rubbing scratch pattern is clearly observed, and then, gives rise to the optical leakage. In this research, to suppress the optical leakage and increase the contrast ratio, we introduce the photo-alignment technique to PSV-FLC.

The materials used in this research were as follows: the FLC was FLC-X (DIC) with a relatively large tilt angle; the photocurable mesogenic monomer was Monomer-A (DIC); and the LC alignment films were polyimide RN-1199 and PHAL-A (Nissan Chemical Industries) for rubbing and photo-alignment technique, respectively. For the photo-alignment, the glass substrates coated with PHAL-A were irradiated with a non-polarized oblique UV light $(254 \mathrm{~nm})$. For the photocure, UV light irradiation $(365 \mathrm{~nm})$ was carried out at the $\mathrm{SmA}$ phase temperature.

Figure 1 shows the microscopic textures of PSV-FLC fabricated in this research. In the PSV-FLC cell fabricated using rubbed RN-1199 alignment films, the rubbing scratch pattern is clearly observed and gives rise to the optical leakage. On the other hand, in the cell fabricated using PHAL-A photo-alignment films, it is found that the more uniform alignment without the rubbing scratch pattern can be obtained. Table 1 shows the optical transmittance of the PSV-FLC cells. It is found that the optical leakage can be largely suppressed in the smectic phase by the photo-alignment technique. Therefore, it is concluded that the photo-alignment technique is more effective to smectic LC devices such as PSV-FLCs.
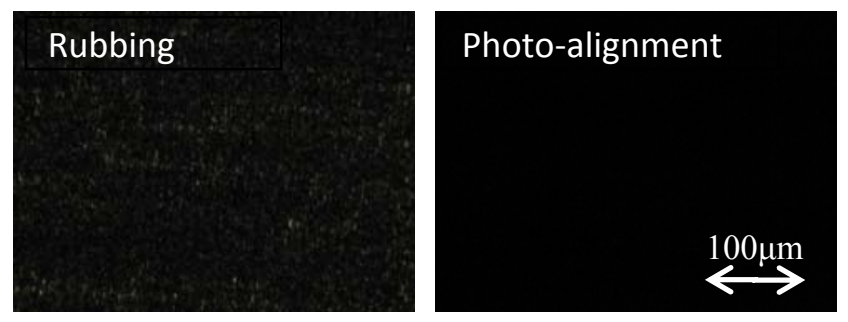

Table 1 Transmittance of PSV-FLCs.

Fig. 1 Microscopic textures of PSV-FLCs.

\begin{tabular}{lcccc}
\hline & \multicolumn{4}{c}{ Transmitance (Arb. u.) } \\
\cline { 2 - 5 } & Iso. & $\mathrm{N}$ & SmA & SmC* \\
\hline \hline Rubbing & 1 & 2.2 & 1.9 & 13.9 \\
Photo-align. & 1 & 4.0 & 1.3 & 7.1 \\
\hline
\end{tabular}

References:

[1] H. Furue et al., Mol. Cryst. Liq. Cryst., 437 (2005) 195.

[2] H. Takahashi et al., Mol. Cryst. Liq. Cryst., 509 (2009) 349.

* presenting author; E-mail: hfurue@rs.noda.tus.ac.jp 